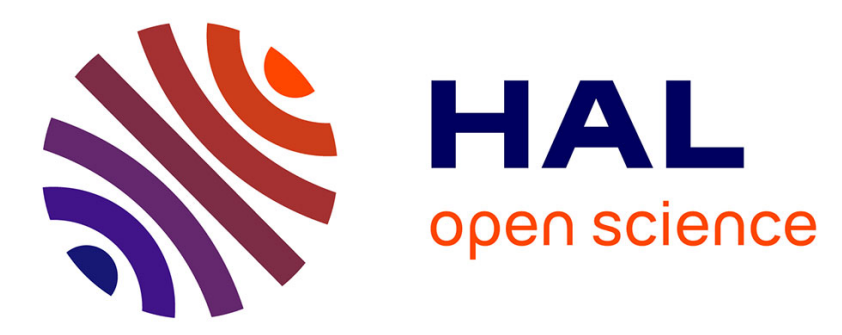

\title{
Comparison of identification protocols of a static hysteresis model
}

\author{
Maria Roberta Longhitano, Fabien Sixdenier, Riccardo Scorretti, Christophe \\ Geuzaine, François Henrotte, Laurent Krähenbühl
}

\section{- To cite this version:}

Maria Roberta Longhitano, Fabien Sixdenier, Riccardo Scorretti, Christophe Geuzaine, François Henrotte, et al.. Comparison of identification protocols of a static hysteresis model. Compumag 2019, Jul 2019, Paris, France. hal-02063610

\section{HAL Id: hal-02063610 https://hal.science/hal-02063610}

Submitted on 22 Jul 2019

HAL is a multi-disciplinary open access archive for the deposit and dissemination of scientific research documents, whether they are published or not. The documents may come from teaching and research institutions in France or abroad, or from public or private research centers.
L'archive ouverte pluridisciplinaire HAL, est destinée au dépôt et à la diffusion de documents scientifiques de niveau recherche, publiés ou non, émanant des établissements d'enseignement et de recherche français ou étrangers, des laboratoires publics ou privés. 


\title{
Comparison of identification protocols of a static hysteresis model
}

\author{
Maria-Roberta Longhitano ${ }^{1}$ Fabien Sixdenier ${ }^{2}$, Riccardo Scorretti ${ }^{1,2}$, \\ Christophe Geuzaine ${ }^{3}$, François Henrotte ${ }^{3}$, Laurent Krähenbühl ${ }^{1}$ \\ ${ }^{1}$ Univ Lyon, ECLyon, Université Claude Bernard Lyon 1, INSA Lyon, CNRS, Ampère, F-69130, Ecully, France \\ ${ }^{2}$ Univ Lyon, Université Claude Bernard Lyon 1, INSA Lyon, ECLyon, CNRS, Ampère, F-69100, Villeurbanne, France \\ ${ }^{3}$ Institute Montefiore - ACE - Université de Liège, B-4000 Liège, Belgium
}

In order to predict magnetic quantities in the magnetic cores of electrical devices, scientists and engineers need hysteresis models sufficiently accurate. Whatever the chosen model is, a dedicated identification protocol has to be elaborated requiring more or less specicfic measurements. This article compares, at least, two identification protocols of the same hysteresis model parameters. The protocols themselves, their inputs and outputs and their accuracy are detailed and discussed.

Index Terms-Magnetic cores, Magnetic devices, Magnetic hysteresis, Magnetic materials,...

\section{INTRODUCTION}

H YSTERESIS models are necessary to predict magnetic quantities in electrical devices for simulation or design purposes. The most used models are the Jiles Atherthon (JA) model [1] and the Preisach model (PM) [2]. One of the last developed model which has a lot of desirable properties is a vector play model (VPM) firstly introduced by Berqvist [3] such as being intrinsically vectorial. Then, pursuing the work, Henrotte showed that its "energy based model" (EBM) provides an accurate energy balance [4] and that the number of cells can strongly improve the accuracy [5]. However, the identification of the model parameters and the number of necessary cells were still issues. That's why two identification protocols concerning this model were proposed in [6] and [7]. The first one is based on an optimization problem where some constraints have been relaxed and a packing algorithm has been proposed. Several objective functions have been tested and gave similar results in terms of accuracy. Moreover this method gave satisfactory results when the temperature has been taken into account. The second one is based on a specific measurement set and the construction of a mathematical function which has to be derived twice in order to obtain the final parameters distribution. In addition, this method gave some qualitative information about the material microstructure (average grain size, density of defects,...) We propose, in this article, to compare these two methods. The paper is organized as follows. The next section will describe, the model and the both identification methods principles. The third section will present the investigated soft magnetic material followed by the measured and simulated results with both methods. Last section is the conclusion.

\section{Model AND IDENTIFICATION METHODS}

\section{A. hysteresis model}

The VPM separates the excitation field $\mathbf{h}$ into a reversible $\mathbf{h}_{r e}$ and an irreversible part $\mathbf{h}_{i r}$ (1)

$$
\mathbf{h}=\mathbf{h}_{r e}+\mathbf{h}_{i r}
$$

To obtain a better accuracy, the model is subdivided into cells. Thus $\mathbf{h}_{r e}$ is a weighted sum of $\mathbf{h}_{r e}^{k}$ contributions of a certain number of cells $N$ (2).

$$
\mathbf{h}_{r e}=\sum_{k=1}^{N} \omega_{k} \mathbf{h}_{r e}^{k}
$$

In (2), $\omega_{k} \geq 0$ is the weight associated with the $k$ cell and must verify $\sum_{k=1}^{N} \omega_{k}=1$.The term $\mathbf{h}_{r e}^{k}$ is the internal state of the cell $k$. It is updated such as (3)

$$
\mathbf{h}_{r e}^{k}= \begin{cases}\mathbf{h}_{r e 0}^{k} & \text { if }\left\|\mathbf{h}-\mathbf{h}_{r e 0}^{k}\right\|<\chi^{k} \\ \mathbf{h}-\chi^{k} \cdot \frac{\mathbf{h}-\mathbf{h}_{r e 0}^{k}}{\left\|\mathbf{h}-\mathbf{h}_{r e 0}^{k}\right\|} & \text { otherwise }\end{cases}
$$

where $\chi^{k}$ is the corresponding maximum pining field of the $k$ cell. The magnetization $\mathbf{M}$ is calculated from $\mathbf{h}_{r e}$ (4).

$$
\mathbf{M}=M_{a n}\left(\left\|\mathbf{h}_{r e}\right\|\right) \cdot \frac{\mathbf{h}_{r e}}{\left\|\mathbf{h}_{r e}\right\|}
$$

Where $M_{a n}\left(\left\|\mid \mathbf{h}_{r e}\right\|\right)$ is a scalar anhystertic function. Finally the flux density $\mathbf{b}$ is obtained with (5)

$$
\mathbf{b}=\mu_{0}(\mathbf{M}+\mathbf{h})
$$

The parameters to identify are then $\left(\omega_{k}, \chi^{k}\right)$. Note that, the $(\omega, \chi)$ distribution can be identified from unidirectional measurements. That's why the vector notations will no longer be present in the document.

\section{B. Identification by optimisation [6]}

The naive way to identify the $\left(\omega_{k}, \chi^{k}\right)$ sets could be such as (6), where the the vectors $Y=\left(\omega_{1} \ldots \omega_{N}\right)$ and $X=\left(\chi^{1} \cdots \chi^{N}\right)$.

$$
\left\{\begin{aligned}
\left(\omega_{k}, \chi^{k}\right)= & \arg \min _{Y, X}\left\|b(h)-b_{s}(h)\right\|_{2}^{2} \\
\omega_{k} \geq 0 & ; \quad \sum_{k=1}^{N} \omega_{k}=1 \\
\chi^{1}=0 & ; \quad \chi^{k} \leq \chi^{k+1}
\end{aligned}\right.
$$

It is proposed to solve (7) instead for reasons detailed after. 


$$
\left\{\begin{array}{l}
\Delta \chi^{k}=\arg \min _{\Delta X}\left\|b(h)-b_{s}(h)\right\|_{2}^{2} \\
\omega_{k} \equiv 1 / N \\
\Delta \chi^{k} \geq 0
\end{array}\right.
$$

Where $\Delta X=\left(\Delta \chi^{1} \ldots \Delta \chi N-1\right)$ and $\Delta \chi^{k}=\chi^{k+1}-\chi^{k}$. With the formulation (7) the problem is "only" a non-negative least square problem. Moreover, (7) has a reduced number of unknowns than (6) $(N-1$ compared to $2 N-1)$. The major improvement is that $\omega_{k}$ is imposed and is no longer a degree of freedom. It is then much easier to solve and avoid of local minima. The price to pay is that a higher number of cells is in principle necessary to obtain a comparable accuracy. That's why, the identification protocol is actually made in two steps. The first step is to solve (7) with a "high" number of cells and then reduce it thanks to a packing cells algorithm shortly described hereafter (more details in [6]).

\section{Identification protocol based on $h_{c}\left(h_{p}\right)$ curve [7]}

In [7], it is mandatory to have the $h_{c}\left(h_{p}\right)$ curve $\left(h_{p}\right.$ is the peak value of an alternating excitation field and $h_{c}$ is the corresponding coercive field) as input data. This measured curve has first to be interpolated and extrapolated as (8). Where $h_{p_{\text {treshold }}}$ is the peak value where $h_{c}$ saturates to its maximum value $h_{c_{\max }}$ when $h_{p}$ goes beyond.

$h_{c}\left(h_{p}\right)= \begin{cases}h_{c}=h_{c_{\max }} & \text { if } h_{p}>h_{p_{\text {treshold }}} \\ h_{c}=\text { interpolation } & \text { if } h_{p_{\min }}<h_{p}<h_{p_{\max }} \\ h_{c}=h_{c_{\min }} \cdot\left(\frac{h_{p}}{h_{p_{\min }}}\right)^{2} & \text { if } h_{p}<h_{p_{\min }}\end{cases}$

Then the auxiliary function $F(h)$ is built: a first point is chosen such as $h>h_{c_{\max }}$ which gives $F(h)=h-h_{c_{\max }}$. The next points are constructed recursively (9) (10)

$$
\begin{gathered}
h^{n}=\frac{h^{n-1}+h_{c}\left(h^{n-1}\right)}{2} \\
F\left(h^{n}\right)= \begin{cases}h^{n}-h_{c_{\text {max }}} & \text { if } h^{n}>h_{p_{\text {seuil }}} \\
\frac{F\left(h^{n-1}\right)}{2} & \text { otherwise }\end{cases}
\end{gathered}
$$

Then $F(h)$ is numerically derived twice 11 which gives a quite continuous $\omega(h)$ distribution. After that, several $\omega_{k}$ and $\chi^{k}$ sets are chosen so as to represent quite accurately the continuous $\omega(h)$ distribution.

$$
\partial_{h} F=\int_{0}^{h} \omega(\chi) d \chi ; \partial_{h}^{2} F=\omega(h)
$$

\section{RESULTS}

In order to avoid any measurement problem, we tested the methods with a virtual material. The virtual material has a Langevin $M_{a n}(h)$ function such as $(12)$, with $M_{s}=1 e 6 \mathrm{~A} / \mathrm{m}$ the saturation magnetization and $h_{0}=10 \mathrm{~A} / \mathrm{m}$ a shape coefficient. The $\omega_{k}$ and $\chi^{k}$ values for the virtual material and those identified by both methods are given in the table 1 .

$$
M_{a n}(h)=M_{s}\left[\operatorname{coth}\left(\frac{h}{h_{0}}\right)-\frac{h_{0}}{h}\right]
$$

TABLE I

REFERENCE AND IDENTIFIED VALUES OF $\omega_{k}$ AND $\chi^{k}$ SETS

\begin{tabular}{|c||c|c|c|c|c|}
\hline Reference & $\omega_{k}$ & 0.1 & 0.4 & 0.3 & 0.2 \\
\hline & $\chi^{k}$ & 0 & 1 & 5 & 15 \\
\hline \hline$\underline{6}$ & $\omega_{k}$ & 0.1667 & 0.333 & 0.3333 & 0.1667 \\
\hline & $\chi^{k}$ & 0 & 0.9865 & 4.8809 & 11.45 \\
\hline \hline$[7 \mid$ & $\omega_{k}$ & 0.22 & 0.521 & 0.226 & 0.04 \\
\hline & $\chi^{k}$ & 0 & 3.17 & 13.83 & 63.32 \\
\hline \hline
\end{tabular}

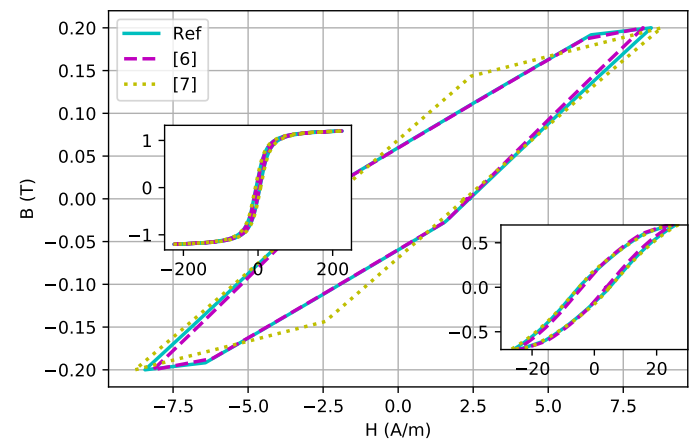

Fig. 1. Loops at different amplitudes. Reference loop (solid line), loops simulated with parameters identified by [6] (dashed line) and loops simulated with parameters identified by [7] (dotted line)

Results show that both methods failed to retrieve "exactly" the parameters sets. The method [6], retrieved quite well the $\chi^{k}$ values (exception for the last value), but the weights are relatively different. The method [7] failed to retrieve both $\chi^{k}$ and $\omega_{k}$. Thus a better accuracy is achieved for loops simulated with parameters identified by [6] than with [7], especially for low amplitude loops.

\section{CONCLUSion}

The extended paper will present more details about the identification methods, more results with real materials and further discussion about accuracy and robustness of each method.

\section{REFERENCES}

[1] D. Jiles and D. Atherton, "Ferromagnetic hysteresis," IEEE Transactions on Magnetics, vol. 19, no. 5, pp. 2183-2185, 1983.

[2] F. Preisach, "ber die magnetische nachwirkung," Zeitschrift fr Physik, vol. 94, no. 1, pp. 277-302, 1935.

[3] A. Bergqvist, "Magnetic vector hysteresis model with dry friction-like pinning," Physica B: Condensed Matter, vol. 233, no. 4, pp. 342-347, 1997.

[4] F. Henrotte, A. Nicolet, and K. Hameyer, "An energy-based vector hysteresis model for ferromagnetic materials," COMPEL, vol. 25, no. 1, pp. 71-80, 2006.

[5] V. Franois-Lavet, F. Henrotte, L. Stainier, L. Noels, and C. Geuzaine, "Vectorial incremental nonconservative consistent hysteresis model," in Proceedings of the 5th International Conference on Advanded COmputational Methods in Engineering (ACOMEN2011). Lige, Belgium: ACOMEN2011, November 2011.

[6] F. Sixdenier and R. Scorretti, "Numerical model of static hysteresis taking into account temperature," International Journal of Numerical Modelling: Electronic Networks, Devices and Fields, pp. e2221-n/a, 2017, e2221 jnm.2221. [Online]. Available: http://dx.doi.org/10.1002/jnm.2221

[7] K. Jacques, S. Steentjes, F. Henrotte, C. Geuzaine, and K. Hameyer, "Representation of microstructural features and magnetic anisotropy of electrical steels in an energy-based vector hysteresis model," AIP Advances, vol. 8, no. 4, p. 047602, 2018. 